Dressings and a bandage around the plaster complete the operation. Antitetanic and anti-gas-gangrene serum are given as a routine. In this manner there is attained an almost perfect apposition of fragments by a relatively simple means and without the inclusion of any foreign material. A successful result depends on the following details:

1. An adequate, but not too generous, excision of the lacerated edge. Removal of wide ellipses of healthy skin is to be avoided, or there will be difficulty in suturing the wound at the end of the operation.

2. Pushing the fragments closely together before the clamp is screwed " home."

3. Using a skin-tight plaster or only one layer of gauze. In a loose plaster the fragments tend to slip out of place. There need be no fear of undue swelling or compression of the limb vessels, for the thickness of the gauze around the clamp, and the presence of the window, provide ample accommodation.

4. Seeing that the foot is only supported under the heel by the flat hand of the assistant. Dorsiflexion above the angle thus produced has a tendency to displace the lower tibial fragment backwards when the bone clamp is removed.

5. Ensuring that the plaster is both thick and dry enough before the clamp is removed.

\section{Further Treatment}

The plaster is not removed for six to eight weeks, depending on the age of the patient. Another smaller one is then applied from toes to just below the knee, and a Böhler " boot",2 incorporated. The patient can walk about in this iron for a few more weeks until the union is firm. In all of the series treated in this way the patients walked unaided and unsplinted in three months. In the most successful case the patient, a boy of 19 , walked at the end of six weeks without stick or splint. At the end of four weeks he had thrown away the bivalve plaster which had been applied with a view to massage, and fortunately had come to no harm. Increasing experience shows that it is better to err on the safe side and leave the plaster on for longer rather than shorter periods, especially as the ambulatory Böhler boot is so comfortable and satisfactory.

The method is only advised for transverse or oblique fractures. The bone clamp, unfortunately, cannot secure sufficient stability in comminuted fractures. In such cases wire extension through the malleoli or os calcis is used, the wound is well washed, and the comminuted fragments. gently pushed into line.

\section{Discussion}

There is no doubt that the one strong objection to this line of treatment is the deliberate laying open of a potentially infected wound and of the manipulation of fragments into line. This criticism will be dealt with.

1. With regard to the reduction of wound sepsis it is cifficult to find out which is the best method. On the one hand certain experienced war surgeons maintain that constant wound irrigation saved numerous limbs. They are supported by Foster, ${ }^{3}$ whose continuous " Dakinization" of 304 consecutive compound fractures warded off sepsis in every case. On the other hand, Winnett Orr and an increasing number of modern surgeons condemn all forms of irrigation, and urge that successful healing will more readily take place if the wound is not inspected for at least six weeks. Such diametrically opposed treatments cannot both be the important factor in reducing sepsis. What is, however, strikingly advocated by both these schools, and indeed by unanimous opinion, is immediate and adequate débridement, followed by complete and absolute immobilization of the limb. The present technique ensures an adequate wound toilet, and provides for complete and absolute rest better than any axis traction or continued tension. Furthermore, the obliteration of gaps, dead spaces, and infected blood clot greatly reduces the bacterial foothold.
2. With regard to the bony union in compound fractures of the lower limb, more than in any other type, it is imperative to have the bony surfaces in as solid a contact as possible. The superiority of this method cannot be denied.

3. With regard to the criticism concerning open manipulation, superficially it would seem that first surgical principles are recklessly disregarded. Is this really so? The treatment of any laceration, by first principles, necessitates the complete excision of all dead material by cutting into healthy tissues immediately surrounding it. The present method carries this principle to excess, and where there is much dead tissue and dirt the débridement-the importance of which has been emphasized by both the irrigation and the non-irrigation schools-can be the more effectively performed. Indeed, in this short series of cases it has always been surprising how much dead tissue, and sometimes dirt, can shelter behind a tiny skin wound. In this method great care is taken of the bones. The main extension is by the assistant at the foot. There is no levering of fragments with elevators or bone spikes, no stripping up of periosteum, or gross manœuvring of any kind. The assistant at the foot simply does what the Kirschner wire would do ; the Lane's forceps guide the fragments into place.

No amount of argument or speculation can decide whether this method and its reasoning is right or not. The cold candour of a long series of cases alone will decide. The severity and frequency of the complications of compound fractures indicate that the best method has not yet been found. The success of the present procedure appears to warrant its further application.

My thanks are due to the surgeons of the West London Hospital and the surgeons of Guy's Hospital for permission to use this method during the years $1929-31$ and 1931-3.

\section{REFERENCES}

${ }^{1}$ Simpson-Smith: British Medical Journal, June 18th, 1932, p. 1133. 2 Böhler: Treatment of Fractures, third edition, p. 158.

3 Foster: Surg., Gynecol. and Obstet., February, 1933, No. 2A, 529.

4 Orr, Winnett: Journ. Bone and Joint Surg., October, 1927, ix, 733 ; ibid., July, 1928, x, 605; Lancet, August 12th, 1933, p. 357.

\section{LUNG ABSCESS FOLLOWING TONSILLECTOMY}

\author{
F. T. RANSON, M.B., B.Ch.BelF., F.R.C.S.ED. \\ AND \\ L. McGOLRICK, M.B., B.CH., B.A.O. N.U.I. \\ SHANGHA \\ (With Special Plate)
}

Lung abscess is a subject more adequately dealt with in American than in British surgical textbooks, and references are more frequent in the American journals. This may be because the disease is reputed to be much commoner in America than in England, and is said to follow one in every 2,500 tonsil operations. ${ }^{1}$ Although tonsillectomy is probably the commonest aetiological factor in lung suppuration, yet some British textbooks do not even mention it in this connexion. Moreover, there is one well-known work which advises the use of an exploring needle for localization. This dangerous and unnecessary practice is almost universally condemned. ${ }^{2}$

\section{CASE RECORD}

The following is an account of a case which we have recently observed.

Miss M., a nursing sister, had a tonsillectomy performed by one of our colleagues on May 17th, 1933. The anaesthetic (ether) was administered by a motor-driven apparatus through 
a Davis-Boyle gag. The patient was a little cyanosed throughout the operation, which was done in the usual manner by dissection and snaring of the pedicle. Haemorrhage was small in amount, and it was not necessary to tie any vessel. A few days after operation the patient developed a cough and a little fever, and later a patch of dullness and crepitations was found at the base of the right lung. The symptoms increased steadily, and by the end of June she had well-marked clinical and radiological signs of lung abscess. She was now very ill, having a severe cough, with foul sputum, and a swinging temperature. A skiagram taken at this time is shown on the Special Plate.

Operation was performed on July 5 th, 1933. The anaesthetic used was $1 / 2$ per cent. novocain, and a little pure chloroform was administered as the ribs were being cut through. An oblique incision was made, with the centre at the posterior axillary line in the eighth interspace; three inches of the eighth and ninth ribs were resected and the intercostal structures and periosteum removed. The parietal pleura was thickened and oedematous, and adherent to the lung over the whole area exposed. The outer wall of the abscess cavity was removed, and about an ounce of foul, stinking pus evacuated. Bands crossing the cavity were not disturbed, and there was very little bleeding. A soft cigarette drain was inserted, and the parietal wound lightly packed with gauze. A transfusion of $500 \mathrm{c} . \mathrm{cm}$. citrated blood was then given. Improvement was immediate; cough, sputum, and fever diminished, and drainage, which was at first free, steadily lessened. Spirochaetes of the Vincent's angina type were found in the pus from the wound, and also in the sputum, and intravenous N.A.B. produced marked local and general improvement. Two weeks after operation the patient developed an effusion into the right pleural cavity. We feared an empyema, but aspiration well away from the wound showed clear fluid, and this absorbed spontaneously in about a week.

August 18th, 1933. Condition as follows: Patient up and walking a little; sinus in chest wall filling in and almost clean. We expect the sinus to be slow in closing. The Special Plate shows an $x$-ray taken a week before this date.

\section{Discussion}

The mode of production of these abscesses is either by embolism or aspiration. We feel certain that in this case the cause was aspiration, and possibly the motor-driven apparatus may have been a factor. Glendenning ${ }^{3}$ condemns the use of motor-driven apparatus in tonsillectomy, but we have employed this apparatus frequently, and know of its use in hundreds of cases with no untoward result.

The question of treatment is difficult. This patient was treated conservatively (postural, inhalations, etc.) for seven weeks. No bronchoscopic drainage was employed, and indeed it is difficult to see how bronchoscopic drainage could benefit a peripheral basal abscess. Young ${ }^{4}$ states that when the condition has been treated medically for six weeks and shows no improvement, then, as a general rule, surgery should be considered ; Lilienthal ${ }^{3}$ is in favour of even earlier intervention. These writers give a full discussion of the indications. Many points have to be considered, and each case must be judged individually. There are three surgical procedures of value: artificial pneumothorax, phrenicectomy, and external drainage. In this case we decided on operation, for the following reasons: (1) After seven weeks' medical treatment the patient was getting much worse. (2) By clinical and radiological investigation we were able accurately to locate the abscess in the periphery of the right lower lobe-a site suitable for external drainage. A valuable sign was present for the few days before operation-namely, a tender spot in the eighth space in the posterior axillary line. It is obyious that artificial pneumothorax would be useless here unless performed in the very earliest stages and before the formation of pleural adhesions. Phrenicectomy we discussed, but as the diaphragm was already raised and fixed on the right side we could see littie object in this operation. On general surgical principles in a case like this external drainage is the obvious and best procedure. Tudor Edwards ${ }^{6}$ advocates that in all cases the two-stage operation should be employed; as we found the lung densely adherent to the parietal pleura, we opened the abscess straight away. In this connexion there is some interesting information in Bickman's Operative Surgery. He quotes various writers, and shows that the artificial production of pleural adhesions by gauze packing or stitching is not so simple as is commonly supposed. The best adhesion-former is the diseased process itself. ${ }^{7}$ Many writers draw attention to the great danger of a coexistent empyema, but Graham $^{8}$ states that all such cases which he has treated recovered after drainage of the pleural cavity only.

Although rare, such a serious complication of tonsillectomy as lung abscess demands the employment of all practical prophylactic measures. For some time now in tonsil cases we have been using slight Trendelenburg position with head extended, so that any blood gravitates to the nasopharynx, and lately we have employed an efficient sucker to keep the pharynx dry. On reading the cases reported in the literature one is struck by the fact that many of them occurred in the hands of very skilled and expert operators, where there was little bleeding and the possibility of such a complication as lung abscess very unlikely.

On the other hand, one has seen cases where blood has accumulated in the lower pharynx-whence it is very likely to be aspirated-and yet no complication has ensued.

We are indebted to Dr. J. E. Bowen for the radiological work.

\section{REFERENCES}

${ }^{1}$ Nelson's Loose Leaf Living Surgery, iv, 5498

2 Ogilvie: Recent Advances in Surgery, p. 161

3 Journ. Amer. Med. Assoc., 1920, lxxiv, 941.

Proc. Roy. Soc. Med., 1932, 1131.

Surg., Gynecol. and Obstet., liii, 788

- Proc. Roy. Soc. Med., 1932, 1131.

"Bickman: Operutive, Surgery, iii, 916.

- Radiology, May, 1933, p. 373 .

\section{A DIVERTICULUM OF THE DUODENUM}

BY

\author{
DAVID CROMIE, M.B., F.R.C.S.ED. \\ SURGEON, NEWTON ABBOT HOSPITAL
}

(With Special Plate)

The condition described below is so rarely met with that an account of a case seen and operated on by the writer may prove of general interest.

A married woman of $\mathbf{3 8}$ had suffered for six months from a severe lumbar pain, which had failed to yield to any ordinary treatment. Two months previously a stabbing pain in the epigastrium supervened, but was overshadowed by the pain in the back. The pain was to the left side of the midline, and did not radiate. It occurred almost immediately after taking food and was relieved by vomiting, after which there was no further pain until the next meal. If no vomiting took place the pain lasted from a half to one hour, and then became easier. The vomiting commenced shortly after the pain in the epigastrium, and took place after almost every meal. Variation of diets made no difference, nor did solids or fluids have any influence. The vomit consisted of undigested food, and was not repeated before the next meal. There had been no haematemesis, nor had there been the periodicity usually associated with ulcer. So far as her appetite was concerned the patient would feel hungry, but "two cream cracker biscuits would make her full up, as if she had been eating all day." The bowels were very constipated. There was no previous history of serious illness, and there was no abnormality of the urine or menstruation, nor did the family history present anything of import. The 


\section{F. T. RANSON AND L. MCGOLRICK: LUNG ABSCESS FOLLOWING TONSILLECTOMY}

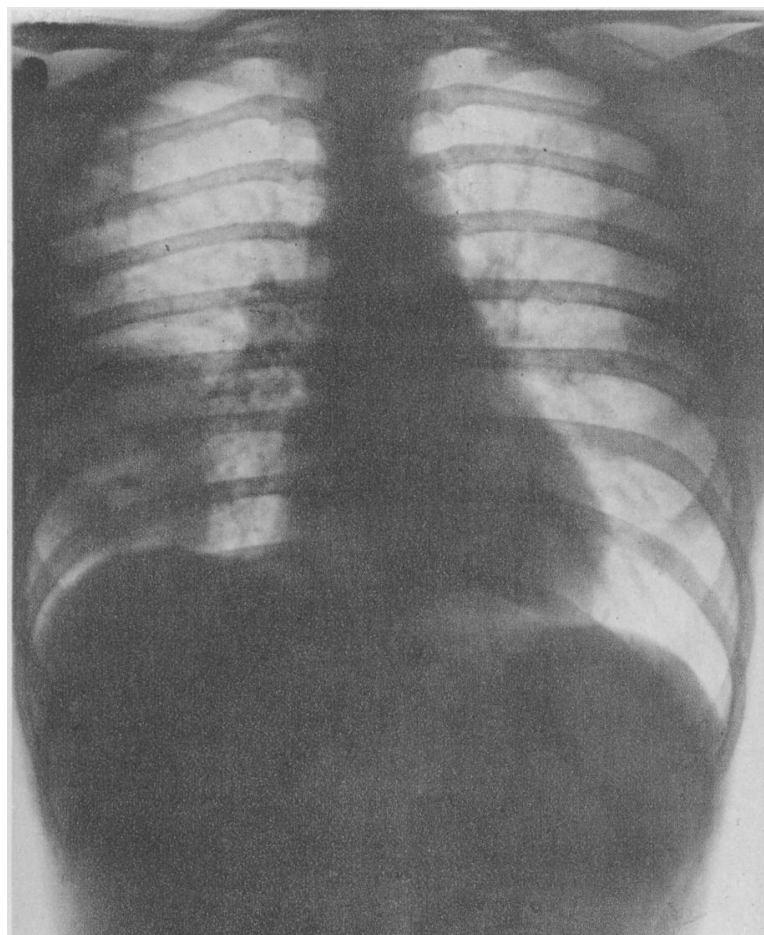

Fio. 1.-Mite M. Showing opacity due to abscess formation at the right base of lung.

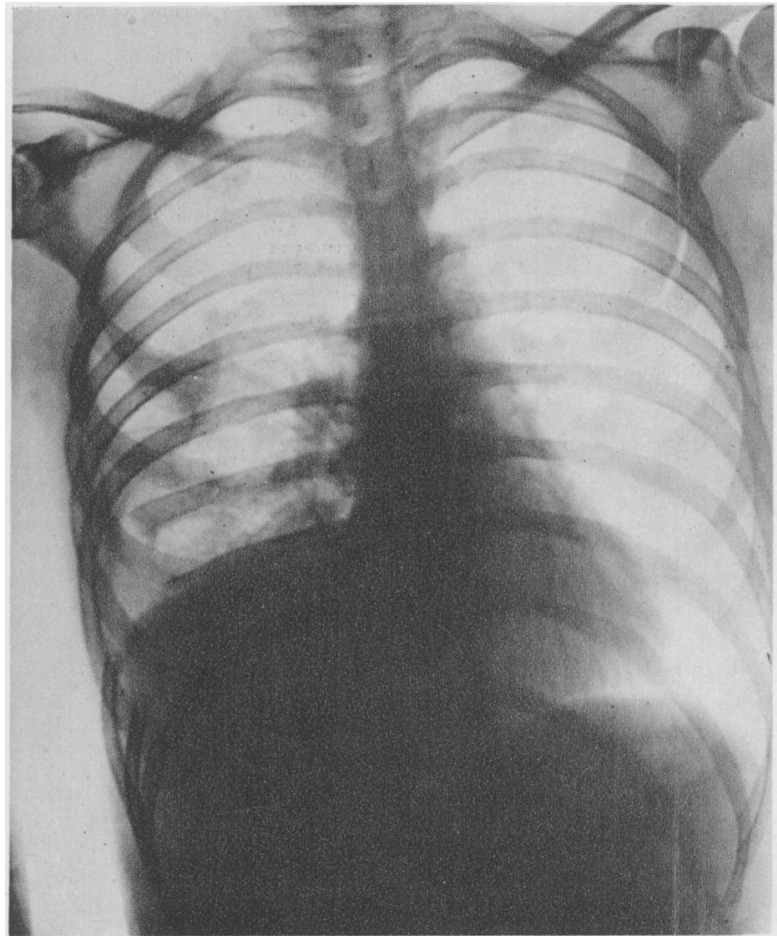

Fia. 2.-The same six weeks later, and more than five weeks after rib resection.
CHÀres E. SALTER: A DIVERTiculum OF THE DUODENUM

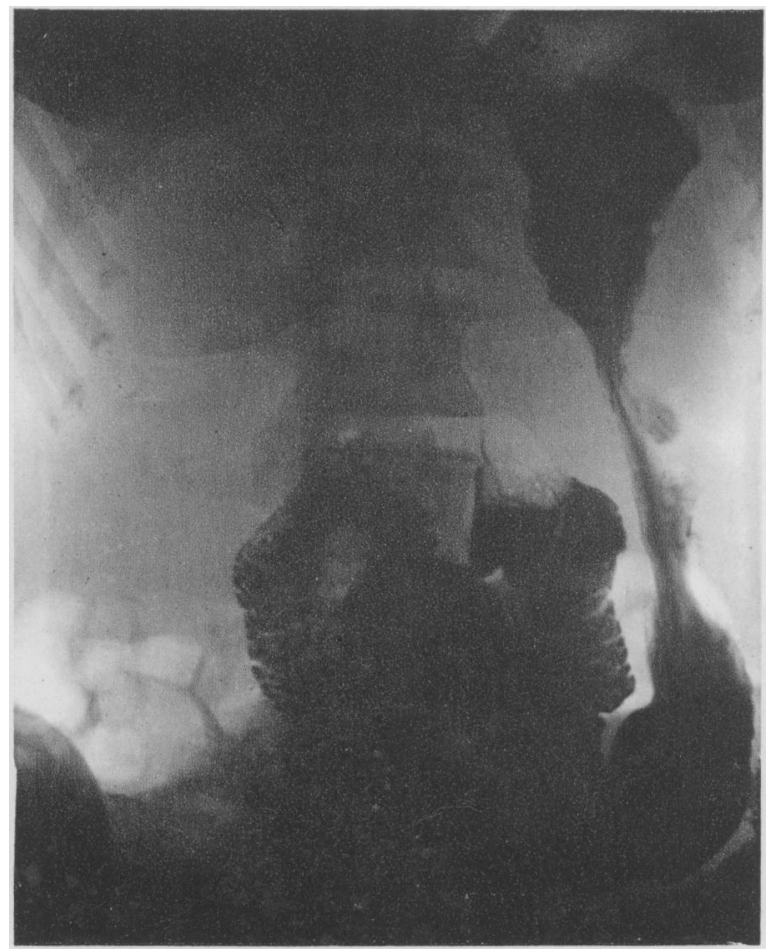

Ten minutes after full ingestion of meal, showing flled diverticulum against body of third lumbar vertebra and a gas bubble above. DUODENUM

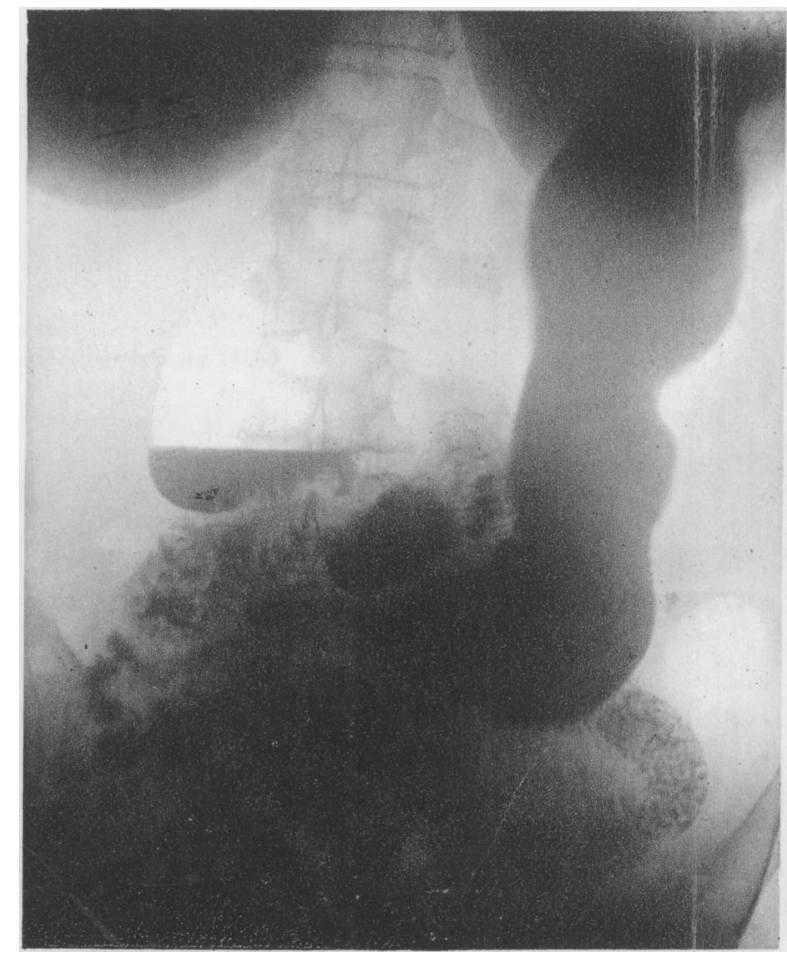

Iarge diverticulum of junction of 1st and 2nd parts of duodenum, showing large aircap with constant fluid level below: appearance the same after three hours. 